\title{
Kilometre-scale, kilowatt average power, single- mode laser delivery through hollow core fibre: overcoming nonlinear limits of glass fibre
}

Hans Christian Mulvad

University of Southampton https://orcid.org/0000-0003-2552-0742

Seyed mohammad Abokhamis Mousavi

University of Southampton https://orcid.org/0000-0002-5250-2630

Viktor Zuba

University of Southampton

Lin Xu

University of Southampton

Hesham Sakr

University of Southampton

Thomas Bradley

University of Southampton

John Hayes

University of Southampton

Gregory Jasion

University of Southampton

Eric Numkam Fokoua

University of Southampton

Austin Taranta

University of Southampton

Shaif-ul Alam

University of Southampton

David Richardson

University of Southampton

Francesco Poletti ( $\square$ frap@orc.soton.ac.uk)

University of Southampton https://orcid.org/0000-0002-1000-3083

Article

Keywords: nested anti-resonant nodeless fibre, continuous wave laser light, laser delivery 
Posted Date: November 1st, 2021

DOI: https://doi.org/10.21203/rs.3.rs-1004375/v1

License: (c) (i) This work is licensed under a Creative Commons Attribution 4.0 International License. Read Full License

Version of Record: A version of this preprint was published at Nature Photonics on May 30th, 2022. See the published version at https://doi.org/10.1038/s41566-022-01000-3. 


\title{
Kilometre-scale, kilowatt average power, single-mode laser delivery through hollow core fibre: overcoming nonlinear limits of glass
}

\section{fibre}

H. C. H. Mulvad ${ }^{1}$, S. Abokhamis Mousavi ${ }^{1}$, V. Zuba ${ }^{1}$, L. Xu' ${ }^{1}$, H. Sakr ${ }^{1}$, T. D. Bradley ${ }^{1}$, J. R. Hayes ${ }^{1}$, G. T. Jasion$^{1}$, E. Numkam Fokoua ${ }^{1}$, A. Taranta ${ }^{1}$, S.-U. Alam ${ }^{1}$, D. J. Richardson ${ }^{1}$, F. Poletti ${ }^{1 *}$

${ }^{1}$ Optoelectronics Research Centre, University of Southampton, Highfield Campus, Southampton, UK.

*e-mail: frap@orc.soton.ac.uk

\begin{abstract}
High power laser delivery with near-diffraction-limited beam quality, widely used in industry for precision manufacturing, is typically limited to tens of metres distances by nonlinearity-induced spectral broadening inside the glass-core delivery fibres. Anti-resonant hollow-core fibres offer not only orders-of-magnitude lower non-linearity, but also loss and modal purity comparable to conventional beam-delivery fibres. Using a single-mode hollow-core nested anti-resonant nodeless fibre (NANF) with $0.74-\mathrm{dB} / \mathrm{km}$ loss, we demonstrate delivery of $1 \mathrm{~kW}$ of near-diffractionlimited continuous wave laser light over an unprecedented 1-km distance, with a total throughput efficiency of $\sim \mathbf{8 0 \%}$. From simulations, more than one order of magnitude further improvement in transmitted power or length should be possible in such air-filled fibres, and considerably more if the core is evacuated. This paves the way to multi-kilometre, $\mathrm{kW}$-scale power delivery - not only for future manufacturing and subsurface drilling, but also for new scientific possibilities in sensing, particle acceleration and gravitational wave detection.
\end{abstract}




\section{Main}

High-speed lithium-ion battery welding, subsurface rock drilling and ultrasensitive interferometric sensors are only some of the applications that could be enhanced or enabled by the long-distance transmission of high average power laser beams with stable spatial, spectral and temporal beam properties. In industrial manufacturing for example, where high-power lasers are widely used for applications such as cutting, welding, marking and additive manufacturing, several key advantages arise from the ability to deliver a near-diffraction-limited laser beam (beam quality factor $M^{2 \sim 1}$ ) to the workbench. The small focal spot sizes possible with such beams allow for higher manufacturing precision, and the associated low beam divergence enables long stand-off distance for remote processing ${ }^{1-3}$. High beam quality is also essential for wobble-welding, a powerful concept that enables higher precision, control and speed in welding processes and also allows the joining of dissimilar materials as required e.g. in battery production for e-mobility ${ }^{2,4,5}$. All such applications dictate a constant drive towards higher laser powers, enabling faster processing times, to be delivered to the workbench from further away, allowing laser source sharing and safer placement of the lasers within larger and larger giga-factories. Transmission of the required high-power beams through a single mode optical fibre is thus highly desirable for safety and reconfigurability ${ }^{6}$. The power output from single-mode laser sources has undergone remarkable progress, driven especially by the development of Ytterbium-doped fibre lasers (YFLs) now reaching continuous wave (CW) single-mode powers up to $10 \mathrm{~kW}^{7}$. However, the transmission of such high-power, low $\mathrm{M}^{2}$ laser beams through conventional single-mode fibres is severely limited by optical nonlinearities in the silica glass forming their core.

Optical nonlinearities in silica fibres include the Kerr effect, stimulated Brillouin scattering (SBS) and stimulated Raman scattering (SRS), the impact of which generally scale in proportion to the propagation length and in inverse-proportion to the mode area ${ }^{8}$. The Kerr effect is mainly a concern for high peak power pulses, where it can lead to self-phase modulation and ultimately self-focusing. 
For CW lasers, the main limitations arise from SBS and SRS, where SBS typically dominates for narrow-linewidth sources, while SRS is the leading effect for kW-level average power sources such as YFLs due to their intrinsic broadband linewidth ${ }^{9,10}$. For example, a standard single-mode fibre (SMF) designed for operation around $1 \mu \mathrm{m}$ has a core diameter of less than $10 \mu \mathrm{m}$ typically, which restricts the transmission of a 1-kW CW single mode beam to well below $10 \mathrm{~m}$ if significant SRS is to be avoided. While various methods for increasing the SRS threshold by suppressing the Stokes wavelength have been shown, more substantial efforts have gone into enlarging the mode area, since this can reduce all the aforementioned silica fibre non-linearities (except self-focusing) ${ }^{10}$. However, the step-index core of an SMF can only be enlarged to approximately $16 \mu \mathrm{m}$ while preserving single-mode (SM) operation and acceptable bend loss, and larger core sizes will lead to multimode guidance ${ }^{3}$. As a strategy to increase the mode area without undesired inter-modal beam instabilities, researchers have turned to alternative fibre designs. Photonic crystal fibres (PCFs) employ a wholly different guidance mechanism and thus have a less constrained relationship between core size and modal content ${ }^{11}$. Chirally-coupled core fibres ${ }^{12}$ and HOM delocalised fibres ${ }^{13}$ introduce loss mechanisms which selectively affect higher order modes (HOMs) to produce effectively SM operation. While such methods have demonstrated effectively SM operation in straight fibres with core diameters exceeding $100 \mu \mathrm{m}$, practical requirements for low bending loss in installations involving flexible and/or bent fibres restrict the core size to values closer to $50 \mu \mathrm{m}$.

Exploiting these ideas enables the transmission of near-diffraction-limited beams with significantly reduced nonlinearity, but few works have demonstrated kW-level power delivery over fibre lengths exceeding a few tens of meters. In ref $^{14}$, fundamental mode propagation in a $60-\mu \mathrm{m}$ core multimode (MM) step-index fibre enabled 100-m transmission of a 1-kW CW laser without onset of SRS, with a beam quality of $\mathrm{M}^{2}=1.3$. Similarly, a $5-\mathrm{kW}$ beam with $\mathrm{M}^{2}=1.3$ was transmitted over a $20-\mathrm{m}$ MM stepindex fibre with a $600-\mu \mathrm{m}^{2}$ effective mode $\operatorname{area}^{15}$. In refs ${ }^{16,17}$, a 3-mode PCF with an effective area of $2500 \mu \mathrm{m}^{2}$ was used to transmit $10 \mathrm{~kW}$ over $30 \mathrm{~m}$ and $1 \mathrm{~kW}$ over $300 \mathrm{~m}$, although at the cost of a somewhat degraded output beam quality in the range $M^{2}=1.7-2.5$ and with the need to impose large 
minimum bend diameters $(\sim 1 \mathrm{~m})$ to ensure acceptable bend losses. Whilst these are impressive achievements, it is evident that single-mode solid-core fibres are operating close to their fundamental beam delivery limits. To transmit these powers over longer distances, or to further increase the delivered power levels, it is essential to look at radically new optical fibre technologies.

\section{Power Delivery in Hollow-Core Fibre}

In hollow-core fibres (HCFs) light travels inside a hollow core surrounded by a silica glass structure ${ }^{18}$. This property can reduce nonlinear impairments to almost insignificant levels and thus enable transmission of average- and peak-power levels far beyond the limitations of silica-core fibres. Research and development of HCFs is currently experiencing significant progress, with a large focus on anti-resonant guiding HCFs (AR-HCFs) ${ }^{19-22}$. The guided mode in this fibre type can have an overlap with the glass structure as low as $10^{-4}-10^{-5}$, which practically eliminates the nonlinear contribution of silica. State-of-the-art AR-HCFs also offer effectively single-mode guidance and ultra-low propagation loss, comparable to or even below the Rayleigh scattering loss limit of silica ${ }^{23-27}$. The unique combination of low loss and negligible nonlinearity offered by AR-HCF presents an as yet unexplored opportunity to transmit ultra-high power levels over long distances without nonlinear signal distortion. Previous works have mostly focused on the transmission of high peak power short pulses over AR-HCF, achieving intensities even higher than the damage threshold of silica, but typically over fibres of a few tens of metres at most ${ }^{28,29}$. Experiments on high average power, neardiffraction-limited CW transmission in AR-HCFs have achieved substantial output power levels ${ }^{30,31}$, even exceeding $1 \mathrm{~kW}^{32}$. However, the propagation lengths demonstrated in these studies were no longer than a few metres - a power and distance combination also achievable with conventional glass fibres.

In this work, thanks to advances in AR-HCF fibre technology, we demonstrate, for the first time, performance well beyond that fundamentally possible in solid core fibres: the transmission of a 1kW average power CW beam with near-diffraction-limited quality over a 1-km long AR-HCF. An input 
coupling efficiency of $95 \%$ and a propagation loss through the fibre of $0.74 \mathrm{~dB} / \mathrm{km}$, comparable to the Rayleigh scattering loss limit of pure silica, enabled nearly $80 \%$ of the laser source power to be delivered at the fibre output in a near-diffraction-limited beam.

\section{Results}

\section{Fibre characterisation}

The AR-HCF used in this work is a 6-element NANF of 1-km length, fabricated using the stack-fuseand-draw method ${ }^{33}$. A scanning electron microscope (SEM) image of the uncoated NANF crosssection is shown in Fig. 1a. The NANF was designed to operate in the $2^{\text {nd }}$ anti-resonance window around $1064 \mathrm{~nm}^{24}$. The core diameter is $31 \mu \mathrm{m}$, the average membrane thickness is $780 \pm 10 \mathrm{~nm}$ and $765 \pm 5 \mathrm{~nm}$ for the inner and outer tubes, respectively, and the cladding diameter is $225 \mu \mathrm{m}$. The fibre is coated with a single $\sim 50 \mu \mathrm{m}$ layer of high-index polymer. The total length of the drawn NANF was $1010 \mathrm{~m}$. However, a small region with defects in the middle of the fibre causing excessive local scattering was identified and cut out, resulting in two separate fibre lengths of $712 \mathrm{~m}$ and $289 \mathrm{~m}$. These were then reconnected by fusion splicing using a standard arc-fusion splicer, with a splice loss estimated to be no more than $0.1 \mathrm{~dB}$. Although the fibre was drawn using inert pressurization gas, free diffusion from the open ends over a period of several months has most likely resulted in its core being filled with a gas mixture close to atmospheric composition during the experiments ${ }^{34}$. Fig. $1 \mathrm{~b}$ shows a cutback spectral loss measurement performed on the resulting 1001-m spliced NANF, together with the simulated total loss and dispersion of the same fibre (cf. Methods). There is very good agreement between the simulated and experimental loss curve in the region 1000-1100 nm. The higher experimental loss around $1120 \mathrm{~nm}$ is attributed to water vapour absorption in the core, which does not impact our experiment and could be purged out if needed. At the laser wavelength of $1075 \mathrm{~nm}$, the propagation loss is $0.74 \pm 0.05 \mathrm{~dB} / \mathrm{km}$. 
a

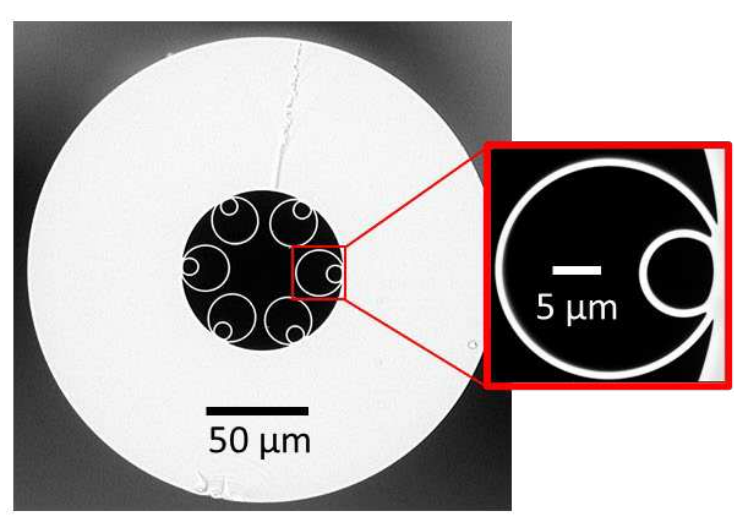

b

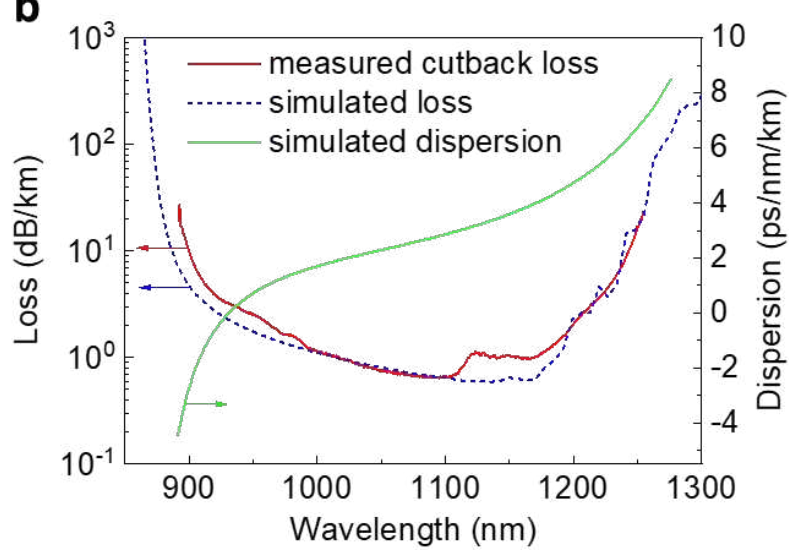

d

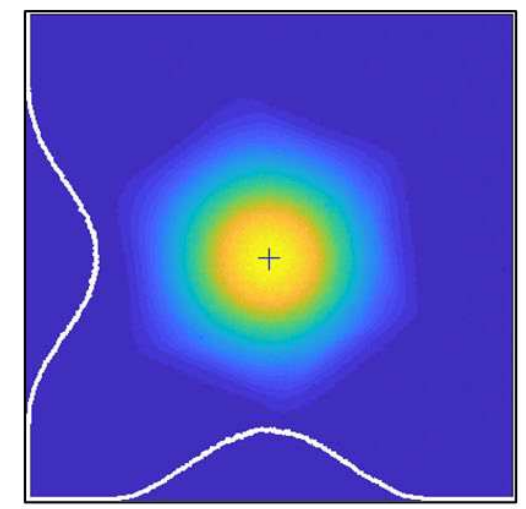

C

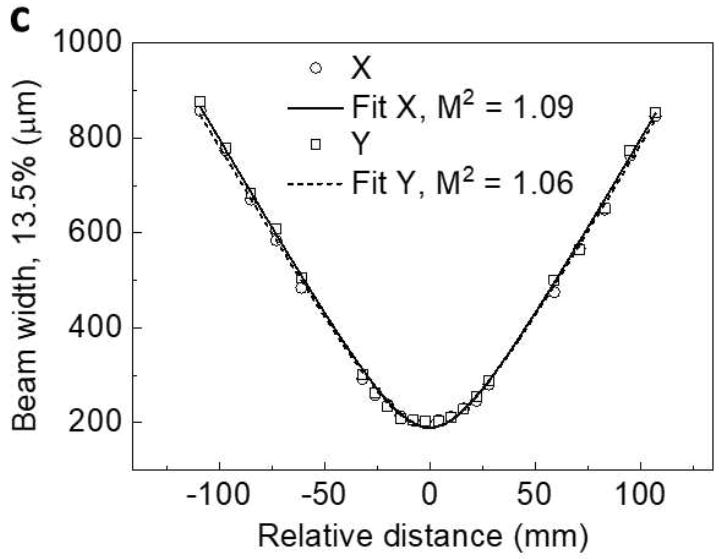

Fig. 1. Characterisation of the 1-km NANF. a Scanning electron microscope image of the fibre cross-section. b Measured and simulated propagation loss (left axis), simulated chromatic dispersion (right axis). $\mathrm{c} \mathrm{M}^{2}$ (beam quality) measurement at $1064 \mathrm{~nm}$. d Near-field camera image of the NANF output beam at 1064nm (horizontal and vertical cross sections through the beam centre are plotted with white lines).

The output beam quality from the 1-km NANF was determined by an $\mathrm{M}^{2}$ measurement in a separate setup using a low-power 1064-nm laser diode (LD) (see Methods). Three consecutive measurements resulted in an $\mathrm{M}^{2}$ value of $1.10 \pm 0.01$ and $1.07 \pm 0.01$ for the horizontal $(\mathrm{x})$ and vertical $(\mathrm{y})$ directions, respectively (one of the measurements is shown in Fig. 1c). This indicates that as a result of the large higher-order mode losses and low intermodal coupling typical of this fibre type ${ }^{21}$, the output light is in the NANF fundamental mode (with mode field diameter of $\sim 22 \mu \mathrm{m}$ ) and has near-diffractionlimited beam quality (a near-field image is shown in Fig. 1d). 
a

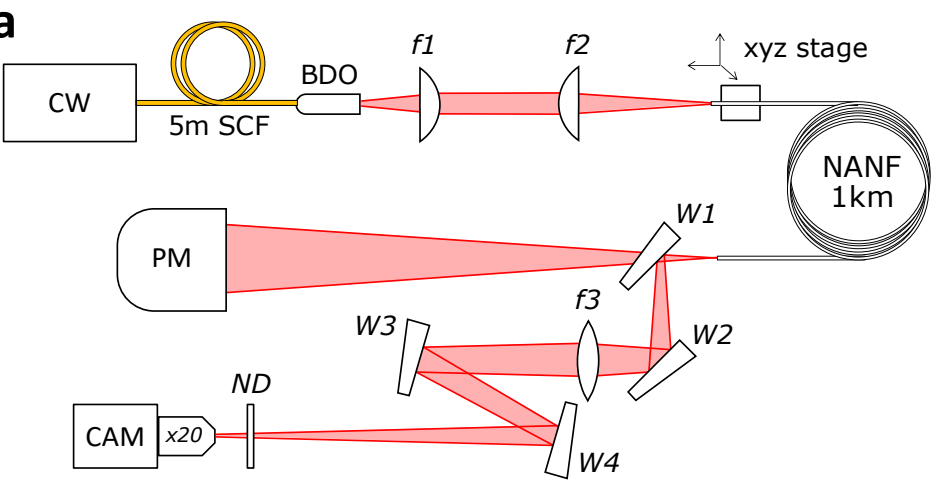

d

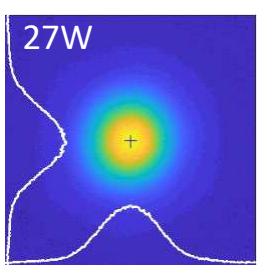

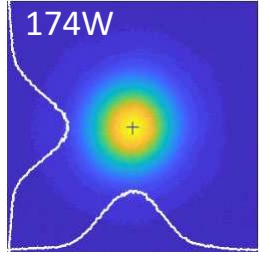

b

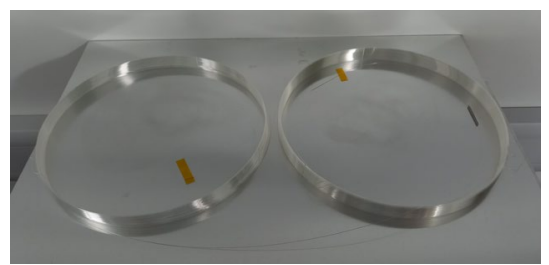

C

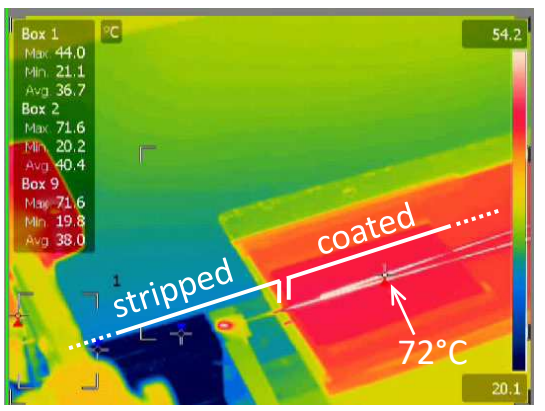

Fig. 2. Demonstration of 1-kW power delivery over the 1-km NANF. a Experimental setup. Abbreviations: CW: continuous wave 1075-nm laser source, SCF: cabled solid-core delivery fibre, BDO: beam delivery optic, f1, f2: plano-convex lenses, f3: double-convex lens, W1, W2, W3, W4: fused silica wedges, PM: power-meter, ND: neutral density filter, $\times 20$ : microscope objective with $\times 20$ magnification, CAM: camera. $\mathbf{b}$ photo of the $1-\mathrm{km}$ NANF. $c$ Thermal camera image of the beginning of the coated NANF fibre following the stripped input section at $P_{\text {in }}=1.38 \mathrm{~kW} . \mathbf{d}$ Near-field camera images (CAM) of the 1-km NANF output beam at three output power levels $\left(\mathrm{P}_{\text {out }}=27 \mathrm{~W}, 174 \mathrm{~W}\right.$ and $\left.1086 \mathrm{~W}\right)$.

\section{Power delivery demonstration}

The experimental setup for the power delivery tests (see Methods) is shown in Fig. 2a, while a photo of the two spliced sections forming the 1-km NANF is shown in Fig. $2 \mathrm{~b}$. The laser source emits a CW beam of wavelength $1075 \mathrm{~nm}$ and beam quality factor $\mathrm{M}^{2 \sim 1}$ 1. The beam is coupled into the NANF under test using two lenses, chosen to achieve the highest possible coupling efficiency (CE) into the fundamental mode of the fibre and thus reduce the risk of thermal damage to the fibre coating from stray light that is not guided in the core. Thermal lensing effects in the coupling optics ${ }^{35}$ were 
compensated by gradually shifting the NANF input tip as the input power was increased, thus enabling maintenance of a high CE (around 95\%) up to power levels well above $1 \mathrm{~kW}$ (see Methods).

The power delivery results for the 1-km NANF can be seen in Fig. 3a. The left axis shows the output power $\left(P_{\text {out }}\right)$ vs the input power into the fibre $\left(P_{\text {in }}\right)$, and the right axis shows the corresponding throughput efficiency $\left(\mathrm{TE}=\mathrm{P}_{\text {out }} / \mathrm{P}_{\text {in }}\right)$. The thermal lensing compensation approach allows a high coupling efficiency to be maintained at all power levels, ensuring a nearly constant TE of almost $80 \%$ (a total loss of $0.97 \mathrm{~dB}$ ). By subtracting the propagation loss of $0.74 \mathrm{~dB}$ as determined from the cutback measurement, a coupling loss of $0.23 \mathrm{~dB}$ is obtained, corresponding to a CE of nearly $95 \%$. This is close to the maximum CE of $\sim 98 \%$ that can be in principle achieved between a Gaussian beam and the mode of a second-window NANF ${ }^{36}$, and is one of the highest CEs reported to date for a hollow core fibre, to the best of our knowledge. The highest output power of $\mathrm{P}_{\text {out }}=1086 \mathrm{~W}$ is obtained for an input power of $\mathrm{P}_{\text {in }}=1377 \mathrm{~W}$, corresponding to a $\mathrm{TE}=79 \%$. The small $1 \%$ drop observable at the highest input powers is likely due to a small input beam distortion from uncompensated thermal lensing. The coating temperature after the initial stripped section of the NANF was monitored using a thermal imaging camera. A linear increase in the temperature vs input power was observed, reaching a stable value of $\sim 72^{\circ} \mathrm{C}$ at the highest input power of $P_{\text {in }}=1377 \mathrm{~W}$, see thermal camera image in Fig. 2c. This temperature is well below the damage threshold of the coating, which should withstand values in excess of $100^{\circ} \mathrm{C}$. For further power scaling beyond this point, improvements to the coupling setup such as use of higher purity lens substrates and cladding light extraction should be adopted, as discussed in Supplementary information. 


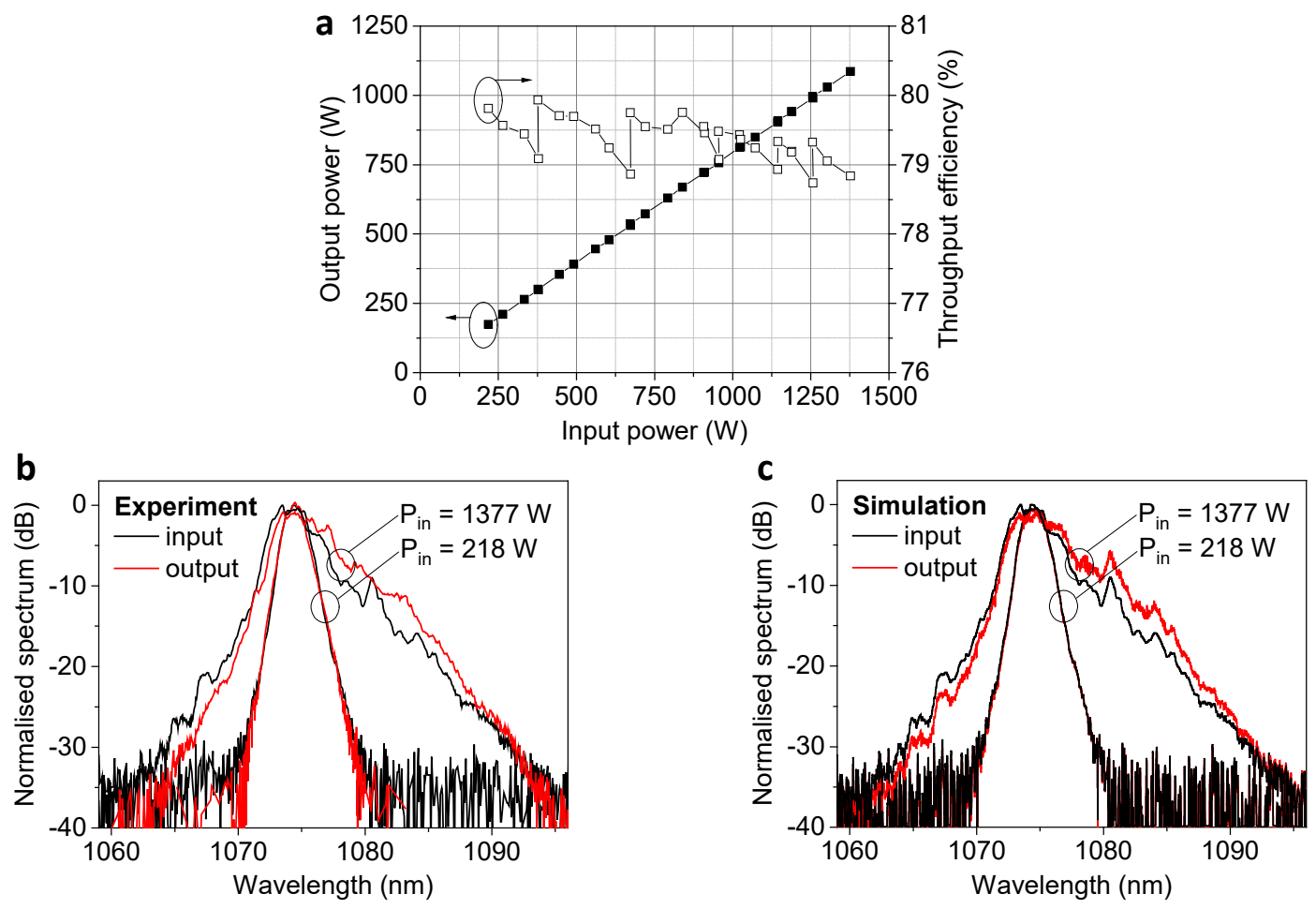

Fig. 3. Power delivery performance of the 1-km NANF. a Left axis: NANF output power $\left(P_{\text {out }}\right)$ vs NANF input power $\left(P_{\text {in }}\right)$. Right axis: throughput efficiency $\left(T E=P_{\text {out }} / P_{\text {in }}\right)$. Note, the step-like increases in $T E$ occur when applying the thermal lensing compensation method. $\mathbf{b}$ Experimentally measured input and output spectra at $P_{\text {in }}=218 \mathrm{~W}\left(P_{\text {out }}=174 \mathrm{~W}\right)$ and $P_{\text {in }}=1377 \mathrm{~W}\left(P_{\text {out }}=1086 \mathrm{~W}\right)$. c Simulated output spectra, based on the same experimentally measured input spectra and $\mathrm{P}_{\text {in }}$ values as shown in $\mathrm{b}$ (plotted also in c for reference).

Fig. 3b shows the output spectrum of the NANF at a relatively low input power level of $\mathrm{P}_{\text {in }}=218 \mathrm{~W}$ $\left(P_{\text {out }}=174 \mathrm{~W}\right)$ and at the highest input power level tested of $P_{\text {in }}=1377 \mathrm{~W}\left(P_{\text {out }}=1086 \mathrm{~W}\right)$. The NANF output spectra are plotted together with the corresponding input spectra (measured separately to the power delivery experiment). Note that the spectral envelope of the laser output increases in width as a function of power, which is attributed to nonlinearity within the laser system itself. Note also that the smaller irregular features on the laser spectrum are not repeatable when turning the laser off and on. As can be seen in Fig. $3 \mathrm{~b}$ for $\mathrm{P}_{\mathrm{in}}=1377 \mathrm{~W}$, a small but noticeable red-shift of the output spectrum ( 1 nm) is observed with increasing power. To investigate its origin, light propagation through the NANF was modelled using the generalised non-linear Schrödinger equation 
(GNLSE), including a semi-quantum model of the rotational and vibrational Raman responses of air (see Methods). The simulated NANF output spectra are shown in Fig. 3c and exhibit a very good agreement with the experimental spectra in Fig. $3 \mathrm{~b}$ at the same power levels. A nearly identical redshift of the spectral envelope can be observed, which our simulations identify as corresponding to the Raman response of the atmospheric air within the core. Due to the small overlap between optical mode and glass membranes (calculated to be $4 \times 10^{-5}$ of the total power for this fibre), the glass nonlinearity is found to play no role at these power levels and distances. We also measured the near-field of the NANF output using the camera and imaging setup shown in Fig. 2a. The recorded mode profiles at $P_{\text {out }}=27 \mathrm{~W}, 174 \mathrm{~W}$ and $1086 \mathrm{~W}$ are shown in Fig. $2 \mathrm{~d}$. No change was observable in the NANF output mode profile as a function of power. Because the measurement setup was situated inside a safety enclosure, it was not practically feasible to carry out an $\mathrm{M}^{2}$ beam quality factor measurement. However, the separate $M^{2} \sim 1.1$ measurement performed using a low-power 1064-nm LD (see Fig. 1c), together with the unaltered mode profile at all power levels in the power delivery test (see Fig. 2d), strongly indicate that a near-diffraction limited output beam quality with a comparable $\mathrm{M}^{2}$-value is achieved also at $1 \mathrm{~kW}$.

\section{Discussion and conclusions}

State-of-the-art AR-HCFs such as NANF are now capable of combining propagation loss comparable to or lower than solid silica fibres in a single transverse mode with negligible nonlinearity. This enables, for the first time, the power delivery of kW-class near-diffraction-limited beams over kilometre length scales, as demonstrated in this work.

To investigate the scalability of this result to even higher power levels or longer transmission distances, we have run numerical simulations under the reasonable assumption that Raman-induced spectral broadening remains the limiting factor (see Methods). An overview of the achievable fibre propagation length vs target output power for the NANF is shown in Fig. 4, where results are also compared to a conventional single mode step-index fibre (SMF) and to a state-of-the-art large core- 
area silica PCF designed for near-diffraction-limited power delivery ${ }^{16,17}$. The diagonal lines indicate the power-distance combination at which half the power is spectrally downshifted outside the original launch signal bandwidth by SRS. As can be seen, our experimental demonstration already represents a $\sim 2.5$ times improvement relative to that fundamentally achievable in the best large effective area glass-core fibres (1 kW over $\sim 400 \mathrm{~m}$ in the PCF). However, this is still well below the ultimate capacity of air-guiding fibres.

For example, our simulations (see Supplementary information) indicate that for a $1 \mathrm{~kW}$ target output power the present NANF could transmit over distances up to $6870 \mathrm{~m}$ with acceptable spectral distortions, 17 times longer than the PCF. At these transmission distances fibre loss starts to play a role, and if one were to use an improved NANF with 5-nested-tube and a measured record-low loss of $0.3 \mathrm{~dB} / \mathrm{km}$ at $1060 \mathrm{~nm}$ (below the Rayleigh scattering loss limit of silica) ${ }^{27}$, the achievable distance would extend to $9640 \mathrm{~m}$. For the transmission of a 10-kW beam, our simulations indicate that more than one kilometre would seem possible in the present NANF ( 30 times longer than the PCF), limited only by Raman scattering in the atmospheric air inside the hollow core. These results would require more sophisticated launching and cladding light extraction techniques (see Supplementary information), but they do seem within the realm of possibility ${ }^{17}$. It is also worth noting that if air were to be evacuated from the core of the fibre, no nonlinear contribution from silica would be observed up to the $10 \mathrm{~kW}$ powers studied here - a potential route to achieving two or more orders of magnitude improvement in power or distance over what is possible with glass fibres. 


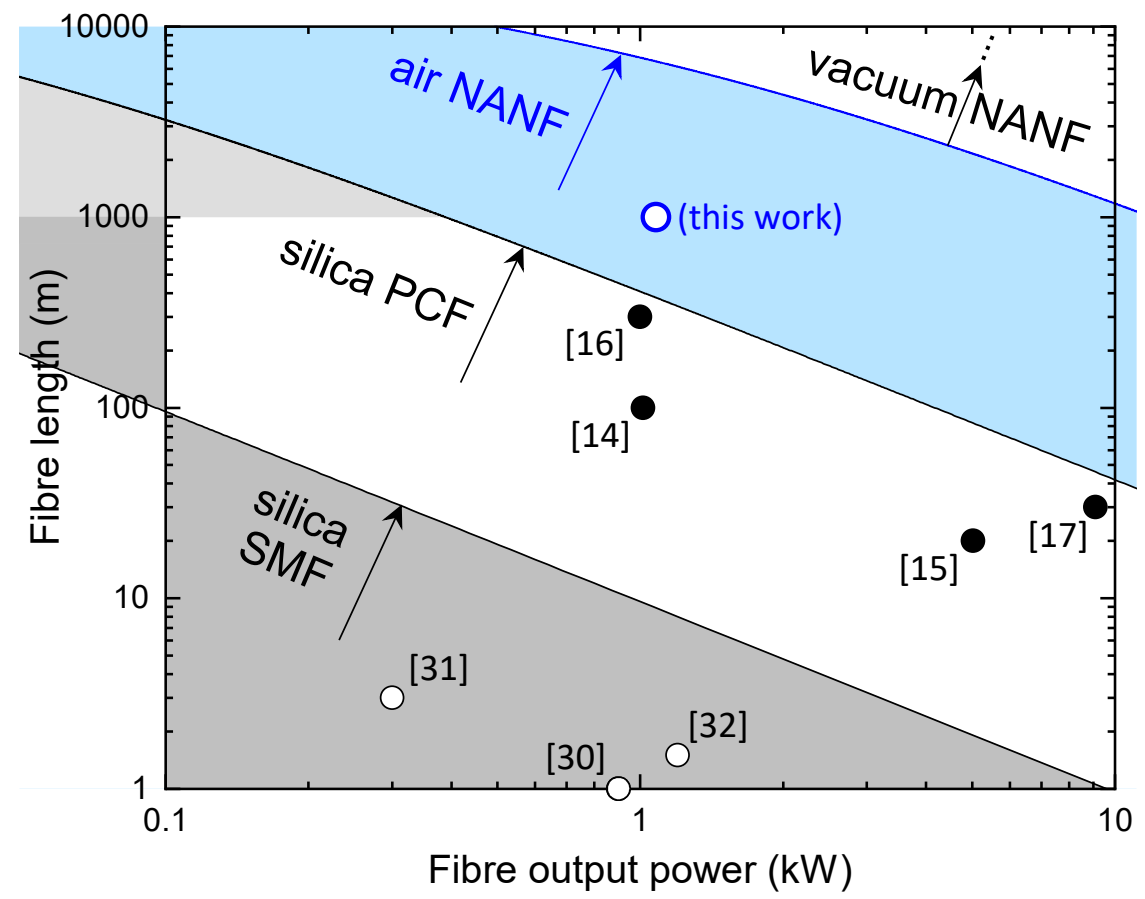

Fig. 4. Scalability of near-diffraction-limited CW power delivery in optical fibres at $1 \mu \mathrm{m}$, limited by stimulated Raman scattering (SRS). The solid lines show the maximum fibre length vs target output power achievable for a standard, commercial step-index silica SMF, a large core-area silica PCF, and an air-filled NANF. Simulations were used to calculate the SRS-induced spectral broadening in both the silica PCF and the air-filled NANF, and the diagonal lines indicate the power-distance combination at which half the power is spectrally downshifted outside the original launch signal bandwidth by SRS. The line for the silica SMF was calculated from the standard approximation for the SRS critical power (see Methods). For a NANF with an empty core (vacuum NANF), no nonlinear limitations were observed in the simulated input power range up to $11 \mathrm{~kW}$, meaning the deliverable output power is only limited by fibre loss. Experimental demonstrations of power delivery including this work are denoted in the figure: silica core fibres (solid circles) and hollow-core fibres (open circles).

The availability of high power, near-diffraction-limited beams allows energy to be optimally directed to improve light-matter interactions, thus increasing energy-efficiency, process-control, functionality and speed. The order(s) of magnitude increase in the distances over which such beams can be delivered through hollow core NANFs can therefore be potentially disruptive in a variety of existing 
and novel applications. For example, NANFs could be deployed to increase the distance between (single-mode) laser and workpiece, offering greater flexibility in the design of production lines and factory floors in future manufacturing ${ }^{37}$. Other laser processing opportunities arise where the target location is hazardous or difficult to access, such as for nuclear decommissioning ${ }^{17,38}$, or in subsurface laser drilling of rocks for oil and gas extraction, where it could provide a safer and more costeffective alternative to the use of charges and hydraulic fracturing, and where multi-kW powers and multi-km distances are required ${ }^{39,40}$.

It might also lead to new scientific opportunities in the trapping and acceleration of particles by radiation pressure. Guidance of particles in HCFs by radiation pressure has already been demonstrated ${ }^{41}$. The possibility of guiding "flying particle sensors" in km-long NANFs could enable sensing of various physical quantities with high positional accuracy at remote locations, e.g. in a radioactive environment ${ }^{42}$. Neutral particle acceleration by radiation pressure, for which Ashkin predicted velocities up to $3 \times 10^{6} \mathrm{~m} / \mathrm{s}$ for micron-sized particles ${ }^{43}$, could be enabled by $\mathrm{km}$-long, vacuum-pumped NANFs, wherein intensity levels could be kept low enough to avoid absorption and evaporation of the accelerated particle. Finally, with some cross-sectional enlargement, it is not unforeseeable that completely straight and vacuum-filled hollow-core fibres might one day be able to support the several hundreds of kW over several kilometres that would make them attractive for long path interferometry, e.g. for gravitational wave detection ${ }^{44}$. 


\section{Methods}

Cutback measurement. For the cutback loss measurement, a free-space launch setup comprising two aspherical lenses was used to couple light into the 1-km NANF. Prior to the cutback procedure, the setup was aligned using a 1064-nm laser diode (LD) with an output power of 10 mW, connected to a PM-980 single-mode patch cord as the launch fibre. The two aspherical lenses were aligned to form an output beam waist matched to the fundamental mode of the NANF, thus achieving an optimised coupling efficiency of approximately $95 \%$ with minimal excitation of higher order modes at $1064 \mathrm{~nm}$. Following this alignment, the input end of the PM-980 patch cord was connected to a white light source (Thorlabs SLS201L/M), thus leaving the launch conditions unchanged. The output of the NANF was then butt-coupled to a 25- $\mu$ m core MM patch cord connected to an optical spectrum analyser (Ando AQ-6315A). After recording the output spectrum of the 1-km NANF, the fibre was cut back to a length of $2 \mathrm{~m}$ while leaving the launch conditions unaltered, and the output spectrum of the 2-m length was recorded. The cutback loss curve in Fig. 1b was obtained by taking the difference between the 1-km NANF and 2-m NANF spectra. Note that the short cutback length was chosen to preserve as much as possible of the length of the 1-km sample. The optimised fundamental mode launch enables a precise estimate of the propagation loss at $1064 \mathrm{~nm}$ even with such a short cutback length. Note however that chromatic aberration in the lenses imply a gradually higher excitation of higher order modes when moving away from $1064 \mathrm{~nm}$, which can contribute to a fundamental mode loss overestimate since the cutback length may be insufficiently long to attenuate the higher order modes. Note also that the PM-980 launch fibre is no longer single-moded below $980 \mathrm{~nm}$, hence exciting higher order modes in the NANF which may similarly lead to an overestimate of the fundamental mode loss (as can be observed in Fig. 1b).

$\mathbf{M}^{2}$ measurement. For the $\mathrm{M}^{2}$ measurement, the same $1064 \mathrm{~nm}$ LD and coupling setup as described above was used to launch light into the 1-km NANF (the input power was $15 \mathrm{~mW}$ ). At the NANF output, a 35-mm focal length plano-convex lens (Thorlabs LA4052-B-ML) was used to collimate the 
beam, followed by a 300-mm focal length plano-convex lens (Thorlabs LA4579-B-ML) to form a focused beam for the measurement. A scanning slit beam profiler (Thorlabs B209-VIS/M) placed on a rail was used to manually measure the beam width as a function of longitudinal distance. The $\mathrm{M}^{2}$ measurement was performed following the method described in standard ISO/TR 11146-3:2004.

Power delivery, coupling setup. A simplified schematic of the power delivery experimental setup is shown in Fig. 2a. The CW laser source is an SPI 2kW Qube Fiber laser emitting at a wavelength of $1075 \mathrm{~nm}$ (model SP-2000-C-W-020-05-PIQ-019-001-000). The laser output is delivered through a cabled $20-\mu \mathrm{m}$ core solid fibre terminated with a water-cooled beam delivery optic (BDO). The BDO and the remainder of the experimental setup is located in an interlocked laser safety enclosure. The BDO output beam divergence is $75 \mathrm{mrad}$ and the beam quality is $\mathrm{M}^{2} \sim 1.1$. The beam is coupled into the NANF via a system of two plano-convex UV fused silica lenses with anti-reflection coating: Thorlabs LA4148-YAG and LA4725-YAG with focal lengths $f_{1}=50 \mathrm{~mm}$ and $f_{2}=75 \mathrm{~mm}$, respectively. The lenses are mounted in low-distortion Polaris-K1F1 mounts from Thorlabs. Note that the focal lengths are chosen so that their ratio is as close as possible to the ratio between the mode-field diameters (MFD) of the delivery fibre ( $\sim 18 \mu \mathrm{m})$ and the NANF fundamental mode ( $22.4 \mu \mathrm{m})$. Gaussian beam calculations are then used to determine the exact positions of the lenses resulting in a beam waist diameter after the second lens equal to the NANF fundamental mode MFD. For example, a waist diameter of $22.4 \mathrm{um}$ is achieved by placing the $\mathrm{f}_{1}$ lens $48.15 \mathrm{~mm}$ after the delivery fibre tip, and the NANF input tip $78.47 \mathrm{~mm}$ after the $\mathrm{f}_{2}$ lens (the distance from the delivery fibre tip to the $f_{2}$ lens is $450 \mathrm{~mm}$ ). The lens mounts are placed on micrometer-controlled translation stages. The built-in red pointing laser of the laser source facilitates lateral alignment of the two lenses to the BDO output beam. The longitudinal positions of the lenses and the NANF input tip are fine-adjusted using the 1075-nm laser output until the NANF output power is maximised. To assist the alignment of the NANF to the incoming beam, scattering from the NANF input facet is monitored using a Thorlabs DCC1645C camera with a 10x microscope objective (positioned at an angle of $\sim 45^{\circ}$ relative to the beam axis). Misalignment of the NANF relative to the incoming beam is clearly visible as 
scattering from the microstructure surrounding the core. For high power launching, the input and output of the NANF are first precision-cleaved using a Fujikura CT-101 fibre cleaver. The first $15 \mathrm{~cm}$ of the input end of the NANF is stripped of its coating and then mounted in a water-cooled aluminium V-groove of length $10 \mathrm{~cm}$. The NANF is fixed to the V-groove using polyimide tape ('Kapton' type). The V-groove is mounted on a Thorlabs MAX381 stepper motor controlled 3-axis stage allowing precise positioning of the NANF input tip. The coupling system was designed to partly compensate for thermal lensing effects ${ }^{35}$ originating from absorption and local heating of the coupling optics at high powers. This manifests itself mainly as a power-dependent reduction in the effective focal length of the lenses, but it is also accompanied by gradual distortion of the beam, both of which, if uncompensated, reduce the CE and increase the risk of coating damage. To compensate for the focal length reduction, as the input power increases the NANF input tip is progressively shifted towards the lens, an approach that proved adequate for maintaining the CE at nearly $95 \%$ up to power levels well above $1 \mathrm{~kW}$.

Power delivery, fibre arrangement. For reasons of fire safety during high power testing, the NANF is not kept on a combustible bobbin (e.g. plastic). Instead, it is rewound at low tension ( $20 \mathrm{~g}$ ) into a free, self-supporting coil of diameter $32 \mathrm{~cm}$ and height $2 \mathrm{~cm}$. This is achieved using a bobbin that can be dismounted after rewinding without affecting the fibre coil. Note that the two lengths of $712 \mathrm{~m}$ and $289 \mathrm{~m}$ constituting the 1-km NANF were wound into separate coils using this method, prior to splicing. While the loss at the splice point is small (estimated to less than $0.1 \mathrm{~dB}$ ), it leads to heat accumulation in the protective splice sleeve, which was therefore kept submerged in water during the power delivery test to dissipate the heat and avoid damage.

Power delivery, output beam characterisation. The output power of the NANF is measured using a water-cooled power meter (PM: Gentech UP55C-2.5KW-HD-D0). The CW laser output power is recorded during the experiment using the internal power meter of the laser source. The corresponding input power to the NANF is determined from a separate reference measurement 
where the PM is placed after the coupling optics, yielding a reference measurement of the NANF input power as a function of the laser internal power meter value. The optical output spectrum of the NANF is measured by picking off some of the scattering from the power meter surface using a multimode fibre, connected to an optical spectrum analyser (OSA: Ando model AQ-6315A). The output mode of the NANF is monitored using the imaging system schematically shown in Fig. 2a. A bi-convex NBK7-substrate lens of focal length $\mathrm{f}_{3}=60 \mathrm{~mm}$ (Thorlabs LB1723-B) relays the near-field image from the end-facet of the NANF output tip to the front image plane of a microscope objective with $\times 20$ magnification (Comar 20/0.4), which then projects the mode image onto the camera detector (Sentech STC-CMB2MPOE-IR). To reduce the power sufficiently for detection by the camera, the NANF output beam is reflected from four fused silica wedges W1-W4 with antireflection coated back-sides to minimise unwanted ghost reflections (W1,W2: Thorlabs BSF20-C; W3,W4: Thorlabs BSF10-C). Wedges W1 and W2 are mounted in a crossed polarisation configuration with a 45-degree angle of incidence. Wedges W3 and W4 are mounted in a co-polarised configuration, with beam incidence angles of $\sim 12^{\circ}$. To further reduce the power level, a neutral density (ND) filter with an optical density of 2.0 (Thorlabs NENIR-20A) is placed after the wedges. The total attenuation of the 4 wedges and ND filter is estimated to about $80 \mathrm{~dB}$, with a polarisationdependent variation of about $1 \mathrm{~dB}$. The camera exposure time is adjusted for each power to stay just below the detector saturation level. Note that the LB1723-B lens $\left(f_{3}\right)$ is placed after W2 to avoid thermal lensing effects (following W2, the power is reduced by $\sim 33 \mathrm{~dB}$ relative to the NANF output). Note also that the transmitted beam through W2 is redirected to the PM using a mirror (this is not shown in Fig. 2a).

Numerical simulations. We used the same approach as $\operatorname{ref}^{45}$ to model the nonlinear light propagation in the air-filled NANF and silica PCF, incorporating a combination of different models and parameters in a unified General nonlinear Schrödinger equation (GNLSE), namely: modeled fiber characterization (i.e. modeled fiber dispersion and total loss), nonlinear model of air and glass, and phase-diffusion model of CW laser. 
The fiber characteristics are modeled using a finite element method (FEM) mode solver (COMSOL) on the geometry of the fiber cross-section extracted from an SEM image for the air-filled NANF. This study provides the required information for nonlinear propagation modeling such as: full chromatic dispersion (material and waveguide dispersion), loss (confinement loss and macro-bend loss) and wavelength-dependent effective mode area. The total modeled loss presented in Fig. $1 \mathrm{~b}$ additionally includes micro-bending loss, which is calculated by the power mode coupling method ${ }^{46}$ applied on micro-bend induced perturbation. The combined total loss is in very good agreement with the measured cutback loss, as shown in Fig. 1b. The simulations indicate that the loss within the antiresonant passband is limited by macro-bending effects towards the shorter wavelengths and confinement loss towards the longer wavelengths.

The silica PCF chosen for our comparison modelling (Fig. 4) is reported in refs ${ }^{16,17}$ for record power delivery. For simplicity, the core, with an effective mode area of $A_{\text {eff }}=2500 \mu m^{2}$, is assumed to support only the fundamental mode, although the actual PCF supports 3 modes. Due to the unavailability of accurate data, the propagation loss and chromatic dispersion were assumed to be dominated by the silica material parameters rather than the waveguide.

For the optical nonlinear response of atmospheric air, we have used a quasi-quantum model which includes an individual model for each gas component (for details and parameters, please refer to ref $\left.{ }^{45}\right)$. The accuracy of this model is also verified independently in ref ${ }^{47}$ for the femtosecond pulse regime. To include the nonlinear response of the glass in the modeling process, we used a multimaterial nonlinear response model by considering the relative power portion in the cladding glass and air core. This method captures the full nonlinear behavior of the fiber in a unified GNLSE, as described in $\mathrm{ref}^{45}$, which enabled us to study the effect of each material (i.e. air or glass) separately and provides accurate modeling results (as shown in Fig. 3b and 3c). 
Modelling a CW laser using the GNLSE is a challenging task since a simple flat-top pulse does not accurately model a realistic CW laser and cannot reproduce the spectral broadening observed in our experiments. As shown in Fig. 3b, the measured spectrum of the CW laser has a broader spectrum in comparison to an ideal $\mathrm{CW}$ laser which is caused by random noise fluctuations ${ }^{48}$. This broadening can be modeled by a well-known phase-diffusion model in the input field envelope ${ }^{49}$ :

$$
E(t)=\sqrt{P(t)} e^{i \delta \phi(t)}
$$

Here, $\delta \phi$ is a phase fluctuation with zero ensemble average and $P$ is the fibre input power of the $\mathrm{CW}$ field. In the frequency domain, $\delta \phi$ manifests itself as random fluctuations, denoted $v_{R}$, of the central frequency of the $\mathrm{CW}$ laser ${ }^{48}$. In the simplest form, such frequency fluctuations $\left(v_{R}\right)$ can be considered as Gaussian white noise with zero mean and a variance of $\sigma_{\nu_{R}}^{2}$ which represents the full width at half maximum (FWHM) spectral linewidth of the CW laser ${ }^{50}$. The phase and frequency fluctuations are related by the following equation ${ }^{48}$ :

$$
\delta \phi(t)=2 \pi \int_{-\infty}^{t} v_{R}(\tau) d \tau
$$

This definition of $v_{R}$ produces a CW field with a Lorentzian power spectrum ${ }^{51}$. Therefore, we adapted the phase-diffusion model to the measured FWHM spectral linewidth of our CW laser and reshaped the spectrum profile of the model to the experimentally measured one while preserving the total power of the laser. This method enabled us not only to include the experimentally measured CW laser in the GNLSE, but also to reproduce the NANF output spectrum with a very good agreement with the experimental results, as shown in Fig. 3b and 3c.

As a measure of spectral broadening applicable to both SRS in an air-filled NANF and SRS in a silicacore fiber, we adopted a "power spectrum overlap ratio" defined as:

$$
\rho(z)=\frac{\int \sqrt{S(0, f) S(z, f)} d f}{\sqrt{\int S(0, f) d f \int S(z, f) d f}} .
$$


Here, $S(z, f)$ is the power spectrum at a propagation distance $z$ and $f$ is the optical frequency. The parameter $\rho(z)$ quantifies the ratio of power transferred outside of the initial spectrum profile. According to this definition, when $\rho(z)$ is 0.5 , the power is equally distributed between the initial spectrum profile and outlier frequencies. This is analogous to the definition of critical power for forward SRS in silica fiber where the power at the pump and Stokes wavelengths are equal, which can be approximated by $P_{c r}=16 A_{\text {eff }} / g_{R} L_{e f f}{ }^{8}$. Here, $A_{\text {eff }}$ is the effective core area, $g_{R}$ is the Raman gain coefficient, and $L_{e f f}=\left(1-\exp \left(-\alpha_{p} L\right)\right) / \alpha_{p}$ is the effective interaction length where $\alpha_{P}$ is the fibre loss at the pump wavelength. Hence, the condition $\rho(z)=0.5$ is used to define the critical - or maximum allowable - spectral broadening in both air-filled NANF and silica-core fibres. This condition is used to calculate and compare the maximum transmission length vs target output power for the fibre types shown in Fig. 4 (diagonal lines). A more detailed explanation of the procedure can be found in Supplementary information. Note that for the silica SMF in Fig. 4, the maximum transmission length was calculated from the above expression for $P_{c r}$ using typical parameters for a standard, commercially available step-index SMF designed for operation around 1 $\mu \mathrm{m}\left(\mathrm{A}_{\text {eff }}=30 \mu \mathrm{m}^{2}, \alpha_{\mathrm{p}}=0.67 \mathrm{~dB} / \mathrm{km}, \mathrm{g}_{\mathrm{R}}=5 \times 10^{-14} \mathrm{~m} / \mathrm{W}\right)$.

\section{Acknowledgements}

The authors gratefully acknowledge support from the European Research Council (ERC) (grant agreement n 682724, 'Lightpipe'), the UK Engineering and Physical Sciences Research Council (EPSRC) (Programme Grant EP/P030181/1, 'Airguide Photonics'), and Saudi Aramco.

SPI is acknowledged for advice, useful discussions and for providing a laser source for initial tests.

Callum R. Smith and Hyuntai Kim are acknowledged for early contributions on the coupling of highpower laser beams into hollow core fibres and for assistance with the splicing of NANF fibres, respectively. Yong Chen is acknowledged for advice and assistance with fibre fabrication. 


\section{Author contributions}

H.C.H.M., V.Z. and L.X. performed the power delivery, loss, and $\mathrm{M}^{2}$ measurements. S.A.M.

performed the simulations. H.S., T.D.B. and J.R.H. fabricated the fibres. G.T.J., E.N.F., and F.P.

designed the fibres. A.T. produced the free-standing NANF coils. H.C.H.M., S.A.M., D.J.R. and F.P.

wrote the manuscript. S.U.A, D.J.R, and F.P. provided overall technical leadership across all aspects of the research.

\section{Competing interests}

The authors declare no competing interests.

\section{References}

1. Zaeh, M. F., Moesl, J., Musiol, J. \& Oefele, F. Material processing with remote technology revolution or evolution? Phys. Procedia 5, 19-33 (2010).

2. Beyer, E., Mahrle, A., Lütke, M., Standfuss, J. \& Brückner, F. Innovations in high power fiber laser applications. in Proc. SPIE 8237, Fiber Lasers IX: Technology, Systems, and Applications. 823717 (SPIE, 2012).

3. Zervas, M. N. \& Codemard, C. A. High Power Fiber Lasers: A Review. IEEE J. Sel. Top. Quantum Electron. 20, 219-241 (2014).

4. Kraetzsch, M. et al. Laser beam welding with high-frequency beam oscillation: Welding of dissimilar materials with brilliant fiber lasers. in International Congress on Applications of Lasers \& Electro-Optics. 169-178 (2011).

5. Schmitt, F. D. et al. Laser Beam Micro Welding With High Brilliant Fiber Lasers. J. Laser Micro Nanoeng. 5, 197-203 (2010).

6. Kratky, A., Schuöcker, D. \& Liedl, G. Processing with kW fibre lasers: advantages and limits. in Proc. SPIE 7131, XVII International Symposium on Gas Flow, Chemical Lasers, and HighPower Lasers. 71311X (SPIE, 2009).

7. Stiles, E. New developments in IPG fiber laser technology. in 5th International Workshop on Fiber Lasers. 4-6 (Fraunhofer IWS, 2009).

8. Agrawal, G. P. Nonlinear Fiber Optics. Third edition, (Academic Press, San Diego, CA, 2001).

9. Dawson, J. W. et al. Analysis of the scalability of diffraction-limited fiber lasers and amplifiers to high average power. Opt. Express 16, 13240-13266 (2008).

10. Jauregui, C., Limpert, J. \& Tünnermann, A. High-power fibre lasers. Nat. Photonics 7, 861-867 (2013).

11. Knight, J. C., Birks, T. A., Cregan, R. F., Russell, P. S. J. \& Sandro, J.-P. d. Large mode area photonic crystal fibre. Electron. Lett. 34, 1347-1348 (1998). <https://digitallibrary.theiet.org/content/journals/10.1049/el_19980965>.

12. Liu, C.-H. et al. Effectively Single-Mode Chirally-Coupled Core Fiber. in Advanced Solid-State Photonics. ME2 (Optical Society of America, 2007).

13. Limpert, J. et al. Yb-doped large-pitch fibres: effective single-mode operation based on higher-order mode delocalisation. Light Sci. Appl. 1, e8 (2012). 
14. Röhrer, C., Codemard, C. A., Kleem, G., Graf, T. \& Ahmed, M. A. Preserving Nearly Diffraction-Limited Beam Quality Over Several Hundred Meters of Transmission Through Highly Multimode Fibers. J. Lightwave Technol. 37, 4260-4267 (2019).

15. Shima, K. et al. 5-kW single stage all-fiber Yb-doped single-mode fiber laser for materials processing. in Proc. SPIE 10512, Fiber Lasers XV: Technology and Systems. $105120 \mathrm{C}$ (SPIE, 2018).

16. Matsui, T. et al. Effective Area Enlarged Photonic Crystal Fiber with Quasi-Uniform Air-Hole Structure for High Power Transmission. IEICE Trans. Commun. E103.B, 415-421 (2020).

17. Okuda, T., Fujiya, Y., Goya, S. \& Inoue, A. Beam Transmission Technology by Photonic Crystal Fiber to Realizes High-precision and High-efficiency Laser Processing Technology. Mitsubishi Heavy Ind. Tech. Rev. 57 (2020).

18. Cregan, R. F. et al. Single-Mode Photonic Band Gap Guidance of Light in Air. Science 285, 1537-1539 (1999).

19. Wang, Y. Y., Wheeler, N. V., Couny, F., Roberts, P. J. \& Benabid, F. Low loss broadband transmission in hypocycloid-core Kagome hollow-core photonic crystal fiber. Opt. Lett. 36, 669-671 (2011).

20. Belardi, W. \& Knight, J. C. Hollow antiresonant fibers with reduced attenuation. Opt. Lett. 39, 1853-1856 (2014).

21. Poletti, F. Nested antiresonant nodeless hollow core fiber. Opt. Express 22, 23807-23828 (2014).

22. Debord, B. et al. Ultralow transmission loss in inhibited-coupling guiding hollow fibers. Optica 4, 209-217 (2017).

23. Gao, S.-f. et al. Hollow-core conjoined-tube negative-curvature fibre with ultralow loss. Nat. Commun. 9, 2828 (2018).

24. Sakr, H. et al. Hollow core optical fibres with comparable attenuation to silica fibres between 600 and 1100 nm. Nat. Commun. 11, 6030 (2020).

25. Gao, S.-f., Wang, Y.-y., Ding, W., Hong, Y.-f. \& Wang, P. Conquering the Rayleigh Scattering Limit of Silica Glass Fiber at Visible Wavelengths with a Hollow-Core Fiber Approach. Laser Photon. Rev. 14, 1900241 (2020).

26. Jasion, G. T. et al. Hollow Core NANF with $0.28 \mathrm{~dB} / \mathrm{km}$ Attenuation in the $\mathrm{C}$ and $\mathrm{L}$ Bands. in Optical Fiber Communication Conference Postdeadline Papers 2020. Th4B.4 (Optical Society of America, 2020).

27. Sakr, H. et al. Hollow Core NANFs with Five Nested Tubes and Record Low Loss at 850, 1060, 1300 and $1625 \mathrm{~nm}$. in Optical Fiber Communication Conference (OFC) 2021. F3A.4 (Optical Society of America, 2021).

28. Debord, B. et al. Multi-meter fiber-delivery and pulse self-compression of milli-Joule femtosecond laser and fiber-aided laser-micromachining. Opt. Express 22, 10735-10746 (2014).

29. Michieletto, M. et al. Hollow-core fibers for high power pulse delivery. Opt. Express 24, 7103-7119 (2016).

30. Hädrich, S. et al. Scalability of components for kW-level average power few-cycle lasers. Appl. Opt. 55, 1636-1640 (2016).

31. Zhu, X. et al. Delivery of CW laser power up to 300 watts at $1080 \mathrm{~nm}$ by an uncooled low-loss anti-resonant hollow-core fiber. Opt. Express 29, 1492-1501 (2021).

32. Palma-Vega, G. et al. High Average Power Transmission Through Hollow-core Fibers. in Laser Congress 2018 (ASSL). ATh1A.7 (Optical Society of America, 2018).

33. Jasion, G. T. et al. Fabrication of tubular anti-resonant hollow core fibers: modelling, draw dynamics and process optimization. Opt. Express 27, 20567-20582 (2019).

34. Rikimi, S. et al. Pressure in As-drawn Hollow Core Fibers. in OSA Advanced Photonics Congress (AP) 2020. SoW1H.4 (Optical Society of America, 2020). 
35. Abt, F., Heß, A. \& Dausinger, F. Focusing of high power single mode laser beams. in International Congress on Applications of Lasers \& Electro-Optics 2007. 202 (Laser Institute of America, 2007).

36. Fokoua, E. N., Slavik, R., Richardson, D. J. \& Poletti, F. Limits of Coupling Efficiency into Hollow-Core Antiresonant Fibers. in Conference on Lasers and Electro-Optics. STu1Q.4 (Optical Society of America, 2021).

37. Zervas, M. N. Bright future for fibre lasers? Laser Systems Europe Winter 2018/2019 (2019). <https://www.lasersystemseurope.com/analysis-opinion/bright-future-fibre-lasers >.

38. Hilton, P. A. \& Khan, A. Underwater cutting using a $1 \mu \mathrm{m}$ laser source. J. Laser Appl. 27, 032013 (2015).

39. Batarseh, S., Gahan, B. C., Graves, R. M. \& Parker, R. A. Well Perforation Using High-Power Lasers. in SPE Annual Technical Conference and Exhibition. SPE-84418-MS (2003).

40. Zediker, M. High power fiber lasers in geothermal, oil and gas. in Proc. SPIE 8961, Fiber Lasers XI: Technology, Systems, and Applications. 89610D (SPIE, 2014).

41. Benabid, F., Knight, J. C. \& Russell, P. S. J. Particle levitation and guidance in hollow-core photonic crystal fiber. Opt. Express 10, 1195-1203 (2002).

42. Bykov, D. S., Schmidt, O. A., Euser, T. G. \& Russell, P. S. J. Flying particle sensors in hollowcore photonic crystal fibre. Nat. Photonics 9, 461-465 (2015).

43. Ashkin, A. The pressure of laser light. Sci. Am. 226, 62-71 (1972).

44. Abbott, B. P. et al. Observation of Gravitational Waves from a Binary Black Hole Merger. Phys. Rev. Lett. 116, 061102 (2016).

45. Mousavi, S. A. et al. Nonlinear dynamic of picosecond pulse propagation in atmospheric airfilled hollow core fibers. Opt. Express 26, 8866-8882 (2018).

46. Marcuse, D. Derivation of Coupled Power Equations. Bell Syst. Tech. J. 51, 229-237 (1972).

47. Luan, J., Russell, P. S. J. \& Novoa, D. Efficient self-compression of ultrashort near-UV pulses in air-filled hollow-core photonic crystal fibers. Opt. Express 29, 13787-13793 (2021).

48. Goodman, J. W. Statistical optics. Wiley classics library edition, (John Wiley \& Sons, New York, 2000).

49. Mussot, A. et al. Spectral broadening of a partially coherent CW laser beam in single-mode optical fibers. Opt. Express 12, 2838-2843 (2004).

50. Cavalcanti, S. B., Agrawal, G. P. \& Yu, M. Noise amplification in dispersive nonlinear media. Phys. Rev. A 51, 4086-4092 (1995).

51. Frosz, M. H., Bang, O. \& Bjarklev, A. Soliton collision and Raman gain regimes in continuouswave pumped supercontinuum generation. Opt. Express 14, 9391-9407 (2006). 


\section{Supplementary Files}

This is a list of supplementary files associated with this preprint. Click to download.

- supplementaryinformation.docx 